\title{
Editorial
}

\section{Leitthema: Benutzer- und Lernermodellierung}

Benutzer- und Lernermodellierung befassen sich mit der Entwicklung interaktiver Computersysteme und insbesondere computerbasierter Lehr-/Lernsysteme. Diese sollen befähigt werden, Annahmen über Eigenschaften individueller Benutzer bzw. Lerner zu bilden, geeignet zu repräsentieren und für die Erzielung eines an den jeweiligen Benutzer bzw. Lerner angepaßten Systemverhaltens zu nutzen. Bei dieser Problemstellung können kognitionswissenschaftliche Erkenntnisse prinzipiell auf zwei Weisen berücksichtigt werden. Zum einen kann es um die Simulation der kognitiven Prozesse eines Benutzers gehen, mit deren Hilfe eine besonders präzise Modellierung angestrebt wird. Zum anderen ist der Prozeß der Benutzer- oder Lernermodellierung selbst ein kognitiver Prozeß, den es auf dem Computer $\mathrm{zu}$ realisieren gilt.

Benutzer- und Lernermodellierung werden also gemeinhin als prototypische Anwendungsgebiete kognitionswissenschaftlicher Theorien und Begriffsbildungen betrachtet. Doch was heißt es eigentlich, Lernende oder (System-)Benutzer zu modellieren? In der Tat gibt es darauf keine eindeutige Antwort. Sowohl Online-Modellierung am laufenden System wie auch eher theoretisch motivierte Simulationsmodelle sind diesem Forschungsbereich zuzuordnen. Auch das Methodeninventar ist vielfältig: Planerkennung, Fehlerdiagnose und Wissensmodellierung sowie reziproke Partnermodellierung im Sinne der Computerlinguistik gehören ebenso dazu wie logikbasierte und probabilistische Verfahren der Wissensrepräsentation, fallbasiertes SchlieBen und auch spezifische Formen maschinellen Lernens. Eine wesentliche Funktion derartiger Modelle, sei es beim theoriebasierten Entwurf oder auch direkt in Unterstützungskomponenten interaktiver Systeme, ist die Adaption des jeweiligen Systems an die Erfordernisse des Benutzers. Dabei ist die Berücksichtigung individueller Unterschiede nur ein Aspekt, die Anpassung an Aufgaben- bzw. Problemkontexte ein mindestens ebenso bedeutsamer.

Läßt sich überhaupt eine verallgemeinernde begriffliche Klammer für diese unterschiedlichen Aspekte finden? Ein allgemeines Prinzip ist wohl das folgende: In einer Situation, in der System und menschlicher Benutzer als (ungleiche) Partner interagieren, geht es darum, das künstliche System mit möglichst viel analytischer Einsicht in die Verhaltensweisen des Gegenübers auszustatten. Dies gilt zumindest im Falle der ,expliziten" oder Online-Modellierung.

Adaptivität und Individualisierung erfahren unterschiedliche Ausprägungen im Rahmen der Lerner- und
Benutzermodellierung. Während bei der allgemeinen Benutzerunterstützung neben der Anpassung von Systemparametern (z. B. an Benutzerpräferenzen, Benutzerkenntnisse oder ein Benutzungsprofil) kontextsensitive Hilfen und flexible Dialogführung im Vordergrund stehen, ist der Anspruch der Lernunterstützung umfassender. Lernermodellierung und Wissensdiagnose sind grundlegende Funktionen intelligenter Lehr-/Lernsysteme, die idealerweise die Rolle eines beliebig verfügbaren und möglichst verständnisvollen Tutors übernehmen sollen. Dieser Anspruch wird nach mehr als zwanzig Jahren der Entwicklung „Intelligenter Tutorieller Systeme" (ITS) heute eher relativiert. Im Gegensatz zu dem ursprünglichen Anspruch ist insbesondere die adaptive, individualisierte Vermittlung neuen Wissens keine Stärke der existierenden ITS im Sinne eines gut ausgearbeiteten, übertragbaren Methodeninventars mit überzeugenden Anwendungsbeispielen. Auch die Berücksichtigung externer sozialer und organisatorischer sowie emotionaler Faktoren ist weiterhin problematisch. Stärken intelligenter lernunterstützender Systeme liegen bei der automatischen Problemlösung und der damit möglichen Hilfestellung, bei der Wissens- und Fehlerdiagnose sowie bei der Generierung von Erklärungen.

Intelligente Lehr-/Lernsysteme bildeten ein zentrales Anwendungsfeld des DFG-Schwerpunktprogramms „Wissenspsychologie". Dies hat mit dazu beigetragen, daß Beiträge seitens der kognitiven Psychologie einen wesentlichen Teil der deutschsprachigen Arbeiten zu ITS über die letzten ca. zehn Jahre ausmachen. Das Thema Benutzermodellierung ist im deutschsprachigen Raum stärker in der Informatik, speziell der KI, sowie in der Computerlinguistik und Software-Ergonomie verankert. Einen besonderen Einfluß hatten hier die Arbeiten des DFG-Sonderforschungsbereichs 314 ,Künstliche Intelligenz und Wissensbasierte Systeme". Die in diesem Themenheft vereinigten Arbeiten spiegeln diese Zuordnungen wie auch charakteristische Forschungsrichtungen wider. Angesichts der Vielfalt von Methoden und Anwendungen müssen jedoch viele einzelne Facetten des gesamten Gebietes ausgeklammert bleiben.

Die Projekte, aus denen in diesem Heft berichtet wird, haben ihre Arbeit in der Regel bereits in zahlreichen internationalen Veröffentlichungen dokumentiert, auf die in den folgenden Beiträgen verwiesen wird. Jedoch enthalten die Artikel dieses Heftes auch jeweils neue Ergebnisse oder beleuchten die Arbeiten aus neuer Sicht. Darüber hinaus sind sie geeignet, die kognitionswissenschaftlich orientierte Forschung zur Benut- 
zer- und Lernermodellierung in Deutschland auch in unserer disziplinär breit gefächerten Leserschaft bekannt zu machen.

Einen Beitrag aus der KI-Forschung, nämlich zu natürlichsprachlichen Dialogsystemen, leisten Ralph Schäfer, Thomas Weis, Thomas Weyrath und Anthony Jameson. Das System READY, an dem sie arbeiten, soll sich in seinem Dialogverhalten an die Ressourcen von Dialogpartnern wie Arbeitsgedächtnis und verfügbare Zeit anpassen. Dieses Problem wird am Beispiel telefonischer Autopannen-Hilfe behandelt. Dieser Beitrag steht beinahe prototypisch für eine bei der Realisierung adaptiver Systeme anzustrebende Herangehensweise: Zum einen werden mächtige KI-Mechanismen zur Repräsentation des Partnermodells und für Schlußfolgerungen über dieses Modell genutzt. In diesem Fall handelt es sich um dynamische Bayessche Netze, die für die Zwecke von READY zu Einflußdiagrammen erweitert werden. Zum anderen wird der Gebrauch dieser Mechanismen durch Bezug auf die kognitionspsychologische Forschung und durch eigene empirische Untersuchungen motiviert.

Einen weiteren bedeutsamen Schritt sind Marcus Specht und Gerhard Weber bei der Entwicklung der Lehr-/Lernsysteme ELM-PE und ELM-ART gegangen. Beide Systeme, die das Erlernen der Programmiersprache Lisp unterstützen, basieren auf der Technik der episodischen Lernermodellierung (ELM), die sich, gemeinsam mit der zugehörigen Inferenzmethode EBR, um die wissenspsychologisch adäquate Modellierung menschlicher Fähigkeiten zur Problemlösung und zum Abruf von Analogien bemüht. So verfolgen ELM und die auf Basis von ELM entwickelten Systeme einen kognitiven Ansatz zur Benutzer- und Lernermodellierung. Die ELM-Methode hat sich gleichermaßen als valide Simulationsmethode und als nützliche Modellierungsmethode zum Einsatz in tutoriellen Systemen erwiesen. Damit liefert dieser Beitrag einen besonders deutlichen Beleg für die Fruchtbarkeit der Verbindung von kognitionswissenschaftlicher Methodik und systemorientierter Benutzer- und Lernermodellierung.

Jörg Schirra behandelt das Thema „Referenzsemantik in der Partnermodellierung" ausgehend von dem konkreten Beispiel einer Sportreportage, bei der die Sprecherrolle des Reporters modelliert wird. Dieses Sprechermodell wird durch ein „Hörermodell" parametrisiert. Das Hörermodell antizipiert Vorverständnis und Erwartungen, vor allem aber die Implikationen des Rezipienten. Aus der Beispielsituation ergibt sich die Fokussierung auf raum-zeitliche Konfigurationen. Ausgehend von Kognitionstheorien, nach denen sich Hörer solche Konfigurationen bildhaft vorstellen, verwendet Schirra die Idee des ,,mentalen Bildes" zur Hörermodellierung. Ein Sprecher berücksichtigt demnach das mentale Bild, das seine Hörer aufgrund seiner Äußerungen vermutlich konstruieren, und die daraus für den Hörer erkennbaren Propositionen. Dieser Vorgang reflektiert die Aktivitäten des Sprechers bei der Bilderkennung und der eigenen Propositionenbildung; die Analogie zwischen äußerem Sehen und innerem Sehen wird hier reizvoll systematisiert.
Während alle bisher erwähnten Beiträge mehr oder weniger direkt mit einem konkreten System (Computermodell) verbunden sind, geht es in dem Beitrag von Josef Lukas um eine vielseitig verwendbare formale Methode der Wissensmodellierung, die u. a. für die Modellierung von Fehlkonzepten bei der Lernermodellierung relevant ist. Ausgehend von einem Ansatz von Doignon und Falmagne formalisiert Lukas „Wissensräume" als algebraische Strukturen. Auf dieser Grundlage können Voraussetzungen von Wissenselementen, bezogen auf bestimmte Aufgabenanforderungen, abgeleitet werden. Diese Voraussetzungen sind im allgemeinen Disjunktionen mehrerer Alternativen, die z.B. verschiedenen möglichen Lösungsmustern entsprechen. Das spezifische Verdienst der Lukasschen Arbeit ist die Erweiterung dieses Ansatzes um die Diagnose und Modellierung von Fehlkonzepten und um die Behandlung von Aufgaben mit mehrwertigen Antwortbewertungen.

Dieses Themenheft enthält also ganz verschiedenartige Beiträge. In den Beiträgen von Schäfer et al. sowie von Specht und Weber werden Arbeiten an konkreten Benutzer- und Lernermodellierungssystemen geschildert, die im Fall des ersten Beitrags als kognitionswissenschaftlich motiviert, im Fall der zweiten Arbeit als kognitionswissenschaftlich fundiert angesehen werden können. Wie Specht und Weber zeigt auch Schirra, daß Partnermodellierung genuin kognitionswissenschaftliche, nämlich in seinem Fall computerlinguistische Fragestellungen aufwerfen kann. Bei Schirra wird diese Fragestellung durch den Entwurf eines Modellierungskonzepts behandelt. Der Artikel von Lukas wiederum trägt ausschließlich zur Methodik insbesondere der Lernermodellierung bei. Diese Ausschließlichkeit ist allerdings durchaus positiv zu bewerten; in der Benutzerund Lernermodellierung sind solche Arbeiten leider viel zu selten zu finden.

Zur Zeit bewegt sich die Forschung zur Benutzerund Lernermodellierung oft auf sehr pragmatischen Pfaden. Nutzerprofile und Nutzungsstatistiken im World-Wide Web und ihre kommerziellen Anwendungen sind zur Zeit von größerem Interesse als kognitiv fundierte Benutzer- und Lernermodelle und dadurch ermöglichte intelligente Anpassungsleistungen in interaktiven Systemen. Die Beiträge dieses Heftes zeigen jedoch, daß Benutzer- und Lernermodellierung und Kognitionswissenschaft in einer engen, wenn nicht wechselseitigen Beziehung zueinander stehen, die weiterhin von Bedeutung sind. Die Beachtung dieser Beziehung sollte in Zukunft sowohl der Verbesserung modellierender Systeme dienen als auch die Breite und Tiefe der kognitionswissenschaftlichen Erkenntnisse zum Thema vergrößern helfen.

Abschließend einige Danksagungen: an Anthony Jameson für die Anregung zu diesem Heft, an Gerhard Strube für sein Einverständnis und seine Unterstützung, an die Gutachter für ihre kompetenten Analysen und hilfreichen Anmerkungen und ganz besonders an die Autoren für die Mühe und Sorgfalt, die sie auf die Erstellung der Manuskripte verwandt haben. Alle haben einen wichtigen Anteil am Zustandekommen dieses Themenheftes. 